\title{
Expression of the spinal 5-HT7 receptor and p-ERK pathway in the carrageenan inflammatory pain of rats
}

\author{
Soo Young Cho, Hyoung Gon Ki, Joung Min Kim, Jin Myung Oh, \\ Ji Hoon Yang, Woong Mo Kim, Hyung Gon Lee, Myung Ha Yoon, and \\ Jeong Il Choi
}

Department of Anesthesiology and Pain Medicine, Chonnam National University Medical School and Hospital, Gwangju, Korea

Background: Although the inhibitory role of the 5-hydroxytrypatmine receptor 7(5-HT7R) on nociceptive processing is generally recognized, an excitatory effect associated with a reduced 5-HT7R expression has also been observed in the nerve injury model. In the carrageenan model, no significant effect is produced by the 5-HT7R activation, but the change in 5-HT7R expression has not been examined. Lesioning of the spinal serotonergic pathway enhances allodynia in the carrageenan model, but it also relieves several other pain states, including in the formalin model. While lesioning suppresses the activation of the extracellular signal-regulated kinase (ERK) of the spinal cord in the formalin model, its role in the carrageenan model has not been reported.

Methods: Following intraplantar injections of carrageenan, the spinal 5-HT7R expression was examined using Western blotting in male Sprague-Dawley rats. The effect of serotonergic pathway lesioning with intrathecal 5,7-dihydroxytryptamine (5,7-DHT) on the expression of the phospho-ERK was measured.

Results: The expression of the 5-HT7R in the carrageenan model was not significantly different from that of naive animals. The expression of the spinal p-ERK in the carrageenan model was significantly increased, but returned to the level of a naive rat 1 hour after the carrageenan injection. However, it remained significantly higher 1 hour after the injection in the animals treated with 5,7-DHT than in the naive and control rats.

Conclusions: The expression of the spinal 5-HT7R is not altered by peripheral inflammation with carrageenan, suggesting that the lack of antinociceptive effect of the 5-HT7R activation is partly attributable to the absence of changes in the expression of the 5-HT7R in the spinal cord. The extended increase of the spinal p-ERK might be related to the enhanced pain behavior in the animals with lesions of the spinal serotonergic pathway in the carrageenan model.

Key Words: 5-HT7 receptor, Carrageenan, Extracellular signal-regulated kinase, Spinal cord.

Corresponding author: Jeong Il Choi, M.D., Ph.D.

Department of Anesthesiology and Pain Medicine, Chonnam

National University Medical School, 42, Jebong-ro, Dong-gu,

Gwangju 501-757, Korea

Tel: 82-62-220-6893, Fax: 82-62-232-6294

E-mail: jichoi@jnu.ac.kr

Received: May 16, 2014.

Revised: 1st, June 11, 2014; 2nd, August 12, 2014; 3rd, August 21, 2014.

Accepted: August 22, 2014.

Korean J Anesthesiol 2015 April 68(2): 170-174

http://dx.doi.org/10.4097/kjae.2015.68.2.170

\section{Introduction}

Serotonin (5-hydroxytryptamine, 5-HT) has been known to play an important role in the modulation of pain at the spinal level through bulbospinal descending projections from the rostroventral medulla [1]. However, the exact role of 5-HT receptor subtypes, including the most recently identified 5-HT receptor 7 (5-HT7R), in both central and peripheral nociceptive processing remains to be elucidated, despite the extensive research available $[2-6]$.

(c) This is an open-access article distributed under the terms of the Creative Commons Attribution Non-Commercial License (http://creativecommons.org/ licenses/by-nc/3.0/), which permits unrestricted non-commercial use, distribution, and reproduction in any medium, provided the original work is properly cited. 
Much evidence has supported the finding that spinal 5-HT7R mediates the inhibitory descending modulation of the nociceptive processing in the spinal cord and the analgesic effect of several drugs including tramadol, morphine or cannabinoids [7-9]. However, it has been noted that the receptor subtypes in the spinal cord on which the 5-HT acts could be involved in determining the role of the descending serotonergic modulation in nociceptive processing at the spinal level $[2,10,11]$. A recent behavioral study demonstrated that the activation of the spinal 5-HT7R with a 5-HT7R agonist had no significant effect on the inflammatory pain induced by an intraplantar injection of carrageenan [4]. In a neuropathic pain model, the expression of the 5-HT7R in the spinal cord was reduced in the animals with neuropathic pain, and the spinal 5-HT7R was assumed to have an excitatory function in nociception [5]. Accordingly, the magnitude of the 5-HT7R expression in the carrageenan model could be lower or equivalent to that of naive animals.

The lack of a significant role has been also reported for another 5-HTR subtype in the carrageenan model. In the case of the 5-HT3 receptor (5-HT3R), a typical 5-HTR subtype for facilitatory modulation, previous studies have shown that the spinal 5-HT3R is actively involved in the inflammatory pain induced by formalin, but that it does not play a significant role in the carrageenan model [12]. As opposed to the carrageenan model, the activation of the 5HT7R was shown to attenuate the pain behavior in the formalin test, suggesting a different role of the 5-HT7R in the two inflammatory pain models [4]. In line with the differential roles of the 5-HT3R and 5-HT7R in the formalin and carrageenan models, lesioning of the serotonergic pathway using a serotonergic neurotoxin (5,7-dihydroxytryptamine, 5,7-DHT) significantly reduced the pain behavior in the formalin test $[13,14]$, but increased the intensity of the mechanical allodynia in the carrageenan model [4]. Interestingly, the reduction of mechanical allodynia with 5,7-DHT pretreatment was also reported in the spinal cord injury model, which goes against the reaction observed in the carrageenan model [15]. In the formalin test, the involvement of the descending serotonergic system in regulating the activation of the extracellular signalregulated kinase (ERK) was demonstrated by showing that the activation of the ERK was reduced by lesioning the spinal serotonergic pathway [14]. Activation of the ERK was also found in the carrageenan rat model [16]. However, the role of the descending serotonergic system in the activation of the ERK has not been examined in the carrageenan model, despite the clear distinction between the two inflammatory pain models regarding the role of the 5-HTR subtypes and the effect of serotonergic pathway lesioning at the spinal level.

This study was designed to test our hypothesis that the 5-HT7R expression of the spinal cord is not different - or even lower - in the carrageenan model, resulting in no antinocicep- tive effect from 5-HT7R activation, and that the lesioning of the serotonergic pathway with 5,7-DHT increases the activation of the spinal ERK, which might be related to the enhanced allodynic response in the carrageenan model.

\section{Materials and Methods}

Male Sprague-Dawley rats weighing 225-250 g were used. All the animals were housed in a room with a constant temperature of $22-23^{\circ} \mathrm{C}$ and an alternating $12 \mathrm{~h}$ light/dark cycle. Water and food were allowed with no limitation. All the experiments were performed according to the International Association for the Study of Pain Guidelines for the Use of Animals in Research.

To induce the inflammatory pain, the animals were injected subcutaneously with $100 \mu \mathrm{l}$ of $2 \%$ carrageenan (degraded $\lambda$-Carrageenan, Wako Pure Chemical Industries, Osaka, Japan) at the center of their hind paw using a 30 gauge needle. The carrageenan was dissolved in saline to form a $2 \%$ solution and was stored at room temperature for 24 hours prior to use.

Two different sets of rats were used for this experiment. One set of animals was injected with carrageenan and the spinal cord was extruded for the measurement of the 5-HT7 receptor in the spinal cord, which was performed 1 and 2 hours after the carrageenan injection. The difference in the expression of the 5-HT7R in the spinal cord was examined between naive (no carrageenan) and carrageenan-injected animals.

The other set of rats was used to examine the changes in pERK in the carrageenan model. The animals were administered with 5,7-dihydroxytryptamine creatinine sulphate salt (5,7DHT, Sigma Aldrich) through an intrathecal (i.t.) catheter for lesioning of the spinal serotonergic pathway. The neurotoxin 5,7-DHT is known to ablate the serotonergic nerve fiber in the spinal cord and to deplete the 5-HT of the spinal cord [15]. Following a previous study [17], a polyethylene-5 (PE-5) catheter connected to an external PE-10 catheter was inserted into the intrathecal space through the atlanto-occipital membrane under general anesthesia. The 5,7-DHT was injected immediately after the insertion of the catheter into the intrathecal space of the lumbar enlargement, and the catheter remained in place for 20 minutes before its removal and skin closure. Desipramine (30 $\mathrm{mg} / \mathrm{kg}$, Sigma Aldrich) was injected intraperitoneally $45 \mathrm{~min}$ before the i.t. injection of 5,7-DHT to prevent the nonspecific uptake of 5,7-DHT by the noradrenergic nerve fibers. The desipramine was dissolved in saline, and the 5,7-DHT was dissolved in saline containing $0.1 \%$ ascorbic acid. Three days after the 5,7DHT injection, the motor function was assessed by testing the righting and placing-stepping reflexes. The pinna reflex and corneal reflex were also examined to evaluate the sensory deficit. I.t. administration of 5,7-DHT $(60 \mu \mathrm{g} / 20 \mu \mathrm{l})$ or vehicle (control) was performed 3 days before the intraplantar injection of the 
carrageenan. The animals were injected with carrageenan and the spinal cord was extruded 30 and 60 minutes after the carrageenan injection to measure the changes in the $\mathrm{p}$-ERK. The changes in the expression of the p-ERK were evaluated in the animals, which had been assigned to one of 3 different treatment groups: naive, vehicle, or 5,7-DHT.

The spinal cord samples obtained as described above were dissected, and the ipsilateral dorsal spinal cord at L4-L6 was extracted, immediately frozen in dry ice, and stored at $-70^{\circ} \mathrm{C}$. After homogenization, centrifugation and determination of the protein concentration of supernatant, equal amounts of protein $(30 \mu \mathrm{g})$ for each sample were loaded and electrophoretically separated, before being transferred to a polyvinylidene difluoride membrane. The primary antibody was a rabbit polyclonal antibody specific to the phospho-ERK (p-ERK, $1: 1,000$, Millipore, Billerica, MA, USA), total-ERK (t-ERK, $1: 1,000$, Millipore, USA), or 5-HT7R ( $1: 1,000$, Novus Biologicals, Littleton, CO, USA). The secondary antibody was a goat anti-rabbit IgG HRPcoupled secondary antibody $(1: 2,000$, Cell Signaling Technology, Danvers, MA, USA). $\beta$-actin was used for the loading control. The immunoreactivity was detected using ECL Western blotting detection reagents (Santa Cruz Biotechnology, Dallas, TX, USA) and then visualized with a LAS-3000 (Life-science, Fujifilm Global, Tokyo, Japan). The data shown was normalized to the values of the naive rat in each blot.
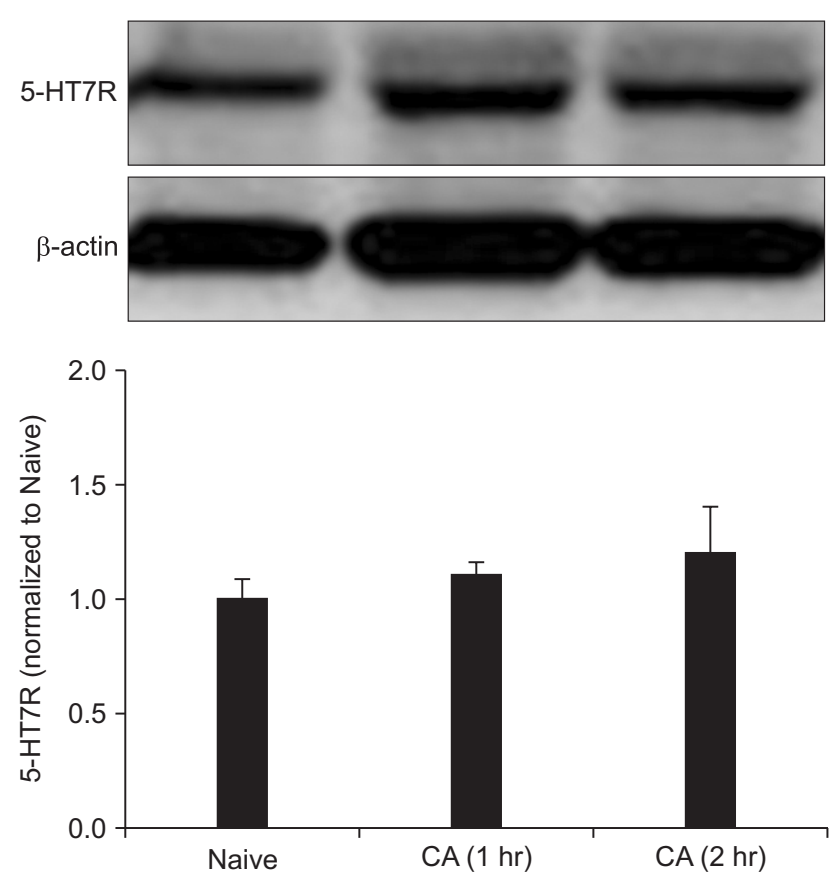

Fig. 1. The level of 5-HT7 receptor does not change following intraplantar injection of carrageenan. Bands of western blotting are representative of that from ipsilateral dorsal spinal cord. No significant differences are observed between naive and carrageenan (CA)-injected animals. $\mathrm{N}=7$ rats/group.
A statistical analysis of the differences among the treatment groups was performed with a t-test or a one-way ANOVA followed by a Bonferroni correction. A value of $\mathrm{P}<0.05$ was considered statistically significant. The data are presented as means \pm standard deviation of mean.

\section{Results}

In Western blotting of the spinal cord, no significant differences were observed between the 5-HT7R expressions before, 1 hour and 2 hours after the intraplantar injection of the carrageenan (Fig. 1).

Intraplantar injection of the carrageenan into the hind paws of the animals elicited a clear threefold increase in the p-ERK at $30 \mathrm{~min}$, compared to naive rat levels. However, it returned to the level of the naive rat at $60 \mathrm{~min}$. In contrast, the animals treated with 5,7-DHT showed a significant increase in the p-ERK at 30
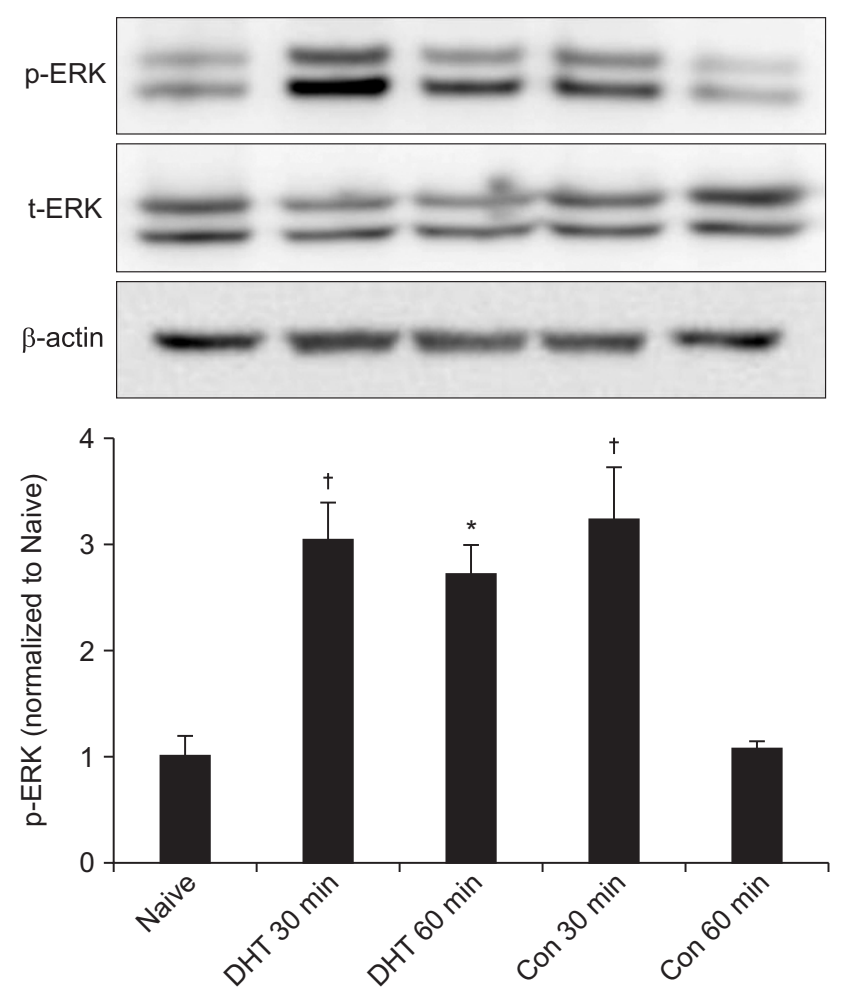

Fig. 2. Representative p-ERK (phospho-extracellular signal-regulated kinase) blots along with t-ERK (total-extracellular signal-regulated kinase) and $\beta$-actin loading controls are illustrated. Intraplantar carrageenan induces a significant increase in p-ERK in dorsal spinal cord. Level of p-ERK increases at $30 \mathrm{~min}$ and returns to the level of naïve at $60 \mathrm{~min}$ after carrageenan injections in control rat. In 5,7-DHT (5,7-dihydroxytryptamine) treated rat, however, increased p-ERK expression is observed at both 30 and $60 \mathrm{~min}$. Densities of t-ERK over $\beta$-actin are not different among groups (data not shown). Con and DHT on abscissa respectively represents the control and 5,7-DHT treated animals, and 30 and $60 \mathrm{~min}$ indicates the time following carrageenan injection. ${ }^{*} \mathrm{P}<0.05,{ }^{\dagger} \mathrm{P}<0.01$, significantly different from Naive. $\mathrm{N}=7$ rats/group. 
$\mathrm{min}$, and it remained elevated at $60 \mathrm{~min}$. The total-ERK expression over $\beta$-actin was not different among the naive and vehicle or 5,7-DHT-injected rats (Fig. 2).

\section{Discussion}

This study demonstrated that the expression of the 5-HT7R is not altered in the carrageenan model, which explains at least in part the lack of antinociceptive effect from a 5-HT7R agonist in the carrageenan model, as observed in a previous report [4].

Contradictory to the inhibitory role of the 5-HT7R, a facilitatory effect was also reported in a neuropathic pain model of spinal nerve ligation in which the 5-HT7R expression in the spinal cord was decreased and the blockade of the 5-HT7R with SB269970 reduced the allodynia and hyperalgesia [5]. Combining this with our results, the overall expression of the 5-HT7R in the spinal cord could be regarded as one of the factors determining the effect of the 5-HT7R activation in the pain models. However, the location and cell type in which the 5-HT receptor is activated may also play an important role in directing the modulation towards inhibition or facilitation [18]. The expression of the 5-HT7R in the spinal cord is mainly found in the superficial lamina of the dorsal horn, specifically in the primary afferent fibers and interneurons of the naive rats [19]. Recent investigation of the antihyperalgesic effect of 5-HT7R activation found that the 5-HT7R is expressed in the GABAergic interneuron of the spinal cord [18]. This finding suggested that the activation of the 5-HT7R on the GABAergic interneurons could potentiate the inhibitory function of the interneuron in spinal nociceptive processing, thereby mediating the antihyperalgesic effect of a 5-HT7R agonist. Accordingly, further investigation on the location and cell type in which the 5-HT7R is mainly activated is warranted to elucidate the underlying mechanisms of the various roles of the spinal 5-HT7R in nociception.

ERK is a member of the mitogen-activated protein kinases family, which transduces a range of extracellular stimuli into intracellular responses [20]. It has also been recognized as a critical player in the development of neural plasticity and pain hypersensitivity [21]. Activation of the ERK in the spinal dorsal horn has been evidenced following nerve injury and peripheral inflammation with formalin, carrageenan, capsaicin or complete Freund's adjuvant $[14,16,22-25]$. In the spinal cord, ERK activation was short-lived and returned to its basal level within 1 to
2 hours after stimulation $[16,26,27]$. However, little is known about the influence of the descending pain modulation mechanism on a marker of nociceptive sensitization such as ERK. The descending serotonergic pathway through the 5-HT3R was also shown to be involved in the activation of the spinal ERK in formalin-induced inflammation [14,21]. Depletion of the spinal serotonin reduced the activation of the ERK evoked by the formalin. It has also been demonstrated that the ERK is activated in the RVM following peripheral inflammation or injury, and much of the ERK activation has been found in the serotonergic neurons $[28,29]$.

Early activation of the ERK in the spinal cord after intraplantar carrageenan injection was reported, suggesting a possible role of the ERK in initiating secondary hyperalgesia [16]. In this study, the activation of the spinal ERK was initiated and dissipated within 1 hour after the injection of the intraplantar carrageenan. A previous study also found early activation of the spinal ERK 30 and $45 \mathrm{~min}$ after the carrageenan injection, which decreased to the level of the naive rat at $90 \mathrm{~min}$, suggesting a role in secondary hyperalgesia around the injection site which develops later than primary hyperalgesia [16]. In the serotonergic pathway-lesioned rats of our study, however, the increase was maintained for up to 1 hour after the carrageenan injection. This finding could be correlated with the enhanced pain following carrageenan inflammation evidenced in a previous report [4], in which the lesioning of the spinal serotonergic pathway using 5,7-DHT enhanced the mechanical allodynia, in particular at the 1 hour mark. Accordingly, a loss of inhibitory descending mechanism via the spinal serotonergic pathway could be responsible for the ERK activation and enhanced pain response after carrageenan inflammation in rats. This finding suggests that the serotonergic modulation has a tonic inhibitory function maintaining the balance between inhibition and facilitation in the inflammatory pain of the carrageenan model.

In conclusion, in support of the lack of antinociceptive effect from a 5-HT7R agonist, the magnitude of the 5-HT7R expression is not significantly changed in the carrageenan model. This study also demonstrates that spinal ERK activation is extended in the case of a lesion in the spinal serotonergic pathway in carrageenan-induced inflammatory pain, suggesting the possibility of a relationship between descending serotonergic modulation and spinal ERK activation.

\section{References}

1. Mason P. Contributions of the medullary raphe and ventromedial reticular region to pain modulation and other homeostatic functions. Annu Rev Neurosci 2001; 24: 737-77.

2. Millan MJ. Descending control of pain. Prog Neurobiol 2002; 66: 355-474. 
3. Suzuki R, Rygh LJ, Dickenson AH. Bad news from the brain: descending 5-HT pathways that control spinal pain processing. Trends Pharmacol Sci 2004; 25: 613-7.

4. Yang J, Bae HB, Ki HG, Oh JM, Kim WM, Lee HG, et al. Different role of spinal 5-HT(hydroxytryptamine)7 receptors and descending serotonergic modulation in inflammatory pain induced in formalin and carrageenan rat models. Br J Anaesth 2014; 113: 138-47.

5. Amaya-Castellanos E, Pineda-Farias JB, Castañeda-Corral G, Vidal-Cantú GC, Murbartián J, Rocha-González HI, et al. Blockade of 5-HT7 receptors reduces tactile allodynia in the rat. Pharmacol Biochem Behav 2011; 99: 591-7.

6. Brenchat A, Nadal X, Romero L, Ovalle S, Muro A, Sánchez-Arroyos R, et al. Pharmacological activation of 5-HT7 receptors reduces nerve injury-induced mechanical and thermal hypersensitivity. Pain 2010; 149: 483-94.

7. Seyrek M, Kahraman S, Deveci MS, Yesilyurt O, Dogrul A. Systemic cannabinoids produce CB(1)-mediated antinociception by activation of descending serotonergic pathways that act upon spinal 5-HT(7) and 5-HT(2A) receptors. Eur J Pharmacol 2010; 649: 183-94.

8. Dogrul A, Seyrek M. Systemic morphine produce antinociception mediated by spinal 5-HT7, but not 5-HT1A and 5-HT2 receptors in the spinal cord. Br J Pharmacol 2006; 149: 498-505.

9. Yanarates O, Dogrul A, Yildirim V, Sahin A, Sizlan A, Seyrek M, et al. Spinal 5-HT7 receptors play an important role in the antinociceptive and antihyperalgesic effects of tramadol and its metabolite, O-Desmethyltramadol, via activation of descending serotonergic pathways. Anesthesiology 2010; 112: 696-710.

10. Bardin L, Bardin M, Lavarenne J, Eschalier A. Effect of intrathecal serotonin on nociception in rats: influence of the pain test used. Exp Brain Res 1997; 113: 81-7.

11. Sommer C. Is serotonin hyperalgesic or analgesic? Curr Pain Headache Rep 2006; 10: 101-6.

12. Rahman W, Suzuki R, Rygh LJ, Dickenson AH. Descending serotonergic facilitation mediated through rat spinal 5HT3 receptors is unaltered following carrageenan inflammation. Neurosci Lett 2004; 361: 229-31.

13. Wei F, Dubner R, Zou S, Ren K, Bai G, Wei D, et al. Molecular depletion of descending serotonin unmasks its novel facilitatory role in the development of persistent pain. J Neurosci 2010; 30: 8624-36.

14. Svensson CI, Tran TK, Fitzsimmons B, Yaksh TL, Hua XY. Descending serotonergic facilitation of spinal ERK activation and pain behavior. FEBS Lett 2006; 580: 6629-34.

15. Oatway MA, Chen Y, Weaver LC. The 5-HT3 receptor facilitates at-level mechanical allodynia following spinal cord injury. Pain 2004; 110: 259-68.

16. Galan A, Lopez-Garcia JA, Cervero F, Laird JM. Activation of spinal extracellular signaling-regulated kinase-1 and -2 by intraplantar carrageenan in rodents. Neurosci Lett 2002; 322: 37-40.

17. Jeong SH, Heo BH, Park SH, Kim WM, Lee HG, Yoon MH, et al. Spinal noradrenergic modulation and the role of the alpha-2 receptor in the antinociceptive effect of intrathecal nefopam in the formalin test. Korean J Pain 2014; 27: 23-9.

18. Viguier F, Michot B, Kayser V, Bernard JF, Vela JM, Hamon M, et al. GABA, but not opioids, mediates the anti-hyperalgesic effects of 5-HT7 receptor activation in rats suffering from neuropathic pain. Neuropharmacology 2012; 63: 1093-106.

19. Doly S, Fischer J, Brisorgueil MJ, Verge D, Conrath M. Pre- and postsynaptic localization of the 5-HT7 receptor in rat dorsal spinal cord: immunocytochemical evidence. J Comp Neurol 2005; 490: 256-69.

20. Pearson G, Robinson F, Beers Gibson T, Xu BE, Karandikar M, Berman K, et al. Mitogen-activated protein (MAP) kinase pathways: regulation and physiological functions. Endocr Rev 2001; 22: 153-83.

21. Ji RR, Gereau RW 4th, Malcangio M, Strichartz GR. MAP kinase and pain. Brain Res Rev 2009; 60: 135-48.

22. Galan A, Cervero F, Laird JM. Extracellular signaling-regulated kinase-1 and -2 (ERK 1/2) mediate referred hyperalgesia in a murine model of visceral pain. Brain Res Mol Brain Res 2003; 116: 126-34.

23. Zhuang ZY, Gerner P, Woolf CJ, Ji RR. ERK is sequentially activated in neurons, microglia, and astrocytes by spinal nerve ligation and contributes to mechanical allodynia in this neuropathic pain model. Pain 2005; 114: 149-59.

24. Adwanikar H, Karim F, Gereau RW 4th. Inflammation persistently enhances nocifensive behaviors mediated by spinal group I mGluRs through sustained ERK activation. Pain 2004; 111: 125-35.

25. Song XS, Cao JL, Xu YB, He JH, Zhang LC, Zeng YM. Activation of ERK/CREB pathway in spinal cord contributes to chronic constrictive injury-induced neuropathic pain in rats. Acta Pharmacol Sin 2005; 26: 789-98.

26. Ji RR, Baba H, Brenner GJ, Woolf CJ. Nociceptive-specific activation of ERK in spinal neurons contributes to pain hypersensitivity. Nat Neurosci 1999; 2: 1114-9.

27. Karim F, Wang CC, Gereau RW 4th. Metabotropic glutamate receptor subtypes 1 and 5 are activators of extracellular signal-regulated kinase signaling required for inflammatory pain in mice. J Neurosci 2001; 21: 3771-9.

28. Geranton SM, Tochiki KK, Chiu WW, Stuart SA, Hunt SP. Injury induced activation of extracellular signal-regulated kinase (ERK) in the rat rostral ventromedial medulla (RVM) is age dependant and requires the lamina I projection pathway. Mol Pain 2010; 6: 54.

29. Imbe H, Kimura A, Okamoto K, Donishi T, Aikawa F, Senba E, et al. Activation of ERK in the rostral ventromedial medulla is involved in hyperalgesia during peripheral inflammation. Brain Res 2008; 1187: 103-10. 